\title{
CAMPO NORMALE E VARIAZIONE SECOLARE MEDIA DEGLI ELEMENTI MAGNETICI IN SICILIA (*)
}

\author{
M. GIORGI - F. Molina
}

Premessa. - Allo scopo di avere una base per il calcolo delle anomalie regionali e per l'interpretazione dei risultati di un rilevamento magnetico della Sicilia, già iniziato nella parte centro-settentrionale dell'isola ( $\left.{ }^{1}\right)$, abbiamo ritenuta opportuna una determinazione del campo normale per le due componenti orizzontale e verticale del campo magnetico terrestre valevole per tutta l'isola.

Per detta determinazione abbiamo preso a fondamento dei calcoli i valori assoluti degli elementi magnetici $D, H, I$ osservati dal$l^{*}$ Istituto Geografico Militare e ridotti al $1935,0\left({ }^{2}\right)$, avendo esclusi i dati di tutte le stazioni anomale.

Dopo il calcolo di una espressione del campo normale di $\mathrm{I}^{\circ}$ grado nelle variabili $\varphi$ (lat. geogr.) e $\lambda$ (long. geogr.), avendo notato che l'approssimazione raggiunta non dava una adeguata rappresentazione di detto campo normale per l'intera isola, abbiamo ripreso i calcoli per determinare una rappresentazione più approssimata di II $^{\circ}$ grado in $\varphi$ e $\lambda$, per entrambe le componenti $H$ e $Z$, che è risultata più soddisfacente.

Avendo poi eseguito una campagna di misure assolute in alcune stazioni, già a suo tempo battute dall'Istituto Geografico Militare, allo scopo di ancorarvi la rete del $\mathrm{II}^{\circ}$ ordine da noi eseguita nella Sicilia centro-settentrionale, abbiamo potuto avere sufficienti dati di osservazione per determinare i valori medi, fra il 1935 e il 1954, della variazione sccolare per gli elementi $D, H$ e $Z$ valevoli almeno per questa zona della Sicilia.

Infatti dal confronto di detti valori con quelli desunti dai dati di osservazione precedenti in Sicilia e con quelli desumibili, per la stessa regione, dai dati planetari di Vestine e collab. $\left({ }^{3}\right.$ ) si trae la conclusione di un soddisfacente accordo e quindi si ha una confortante conferma della attendibilità dei valori ottenuti.

(*) Comunicazione presentata allia (Association internationale de Magnétisme et electricité terrestre" nella $X$ Assemblea generale dell'L.G.G.I. - Roma 1951. 
"Campo normale" delle componenti $I I$ e Z per la Sicilia. - Per il calcolo del "campo normale" relativo alle due componenti $H$ e $Z$ del c.m.t. sono stati utilizzati i dati topografici e magnetici delle osservazioni eseguite dall letituto Gconrafico Militare, riportati in un lavoro di C. Morelli $(\stackrel{2}{)}$.

Sono stati presi in esame i dati degli elementi magnetici di tutte le 145 stazioni eseguite in Sicilia dall'J.M. allo scopo di climinare tutte le stazioni da ritenersi anomale e sono state infine utilizati solo i dati di 81 stazioni.

Nella tabella n. 1 rengono numerate le stazioni da noi prescelte con le indicazioni delle localitì e dei numeri delle corrispondenti stazioni dell I.G.M. riferite nella nota $i^{-1}$ q qià citata, nonchi le indicazioni del quadrante del foglio della carta alla scala 1:100.1100 in cui trovansi ubicate.

Per i lati magnetici e topografici si rinvia alla stessa nota.

Per ogni stazione assoluta è stato calcolato il valore della $Z$ corrispondente mediante i ralori di $H$ e $I$ con la relazione: $Z=I I \operatorname{tg} I$.

Sono state dapprima ealcolate, mediante il metodo dei minimi quadrati, delle espressioni di $1^{\circ}$ grado in $p$ e $\lambda$ per entrambe le componenti $I I$ e $Z$, ma poiché gli scarti tra i valori ottenuti con queste espressioni e i valori osservati di $I I$ e $Z$ erano troppo grandi e superavano la precisione delle singole misure abbiamo dovuto ricorrere a espressioni di $\mathrm{II}^{\circ}$ grado del campo normale.

Per le funzioni di $I^{\circ}$ grado del campo normale sono stati ottenuti infatti i seguenti valori degli errori medi dell'unità di peso:

$$
\mu_{\mathrm{II}}= \pm 32,1 \gamma \quad \mu_{z}=79,5 \gamma
$$

Sono state quindi costruite 81 equazioni di $\mathrm{II}^{\circ}$ grado in "? e i. del segruente tipo per ciascuma delle due componenti $H$ e $Z$ :

$$
T\left(\varphi_{i}^{\prime}, \lambda_{i}^{\prime}\right)=A+B \varphi_{i}^{\prime}+C{\lambda_{i}^{\prime}}^{\prime} D \varphi_{i}^{\prime 2}+E{\lambda_{i}^{2}}^{2}+F \varphi_{i}^{\prime} \lambda_{1}^{\prime}
$$

dove $T$ è la componente del c.m.t. considerata, $\mathcal{Q}_{i}^{\prime} \grave{e}$ la differenza espressa in primi tra la latitudine geografica $\varphi_{i}$ della stazione $i^{\text {ma }}$ e la lat. di riferimento $\varphi_{0}=36^{\circ} \mathrm{N}, \lambda_{1}^{\prime}$ è invece la longit. geogr. della stessa stazione rispetto al meridiano di M. Mario espressa ugualmente in primi.

Applicando alle [1] il metodo dei minimi quadrati per trovare i valori più probalıili delle incognite $A, B, \ldots F$, sono stati ottenuti $\mathrm{i}$ 
seguenti sistemi risolventi di Gauss rispettivamente per $H$ e per $Z$ : $81 \mathrm{~A}+8289.9 \mathrm{~B}+6.560,1 \quad \mathrm{C}+886923,01 \quad \mathrm{D}+730319,15 \quad \mathrm{E}+6.65 .992,53 \quad \mathrm{~F}=$ $=207+238$

$38.498,40 \mathrm{~B}-16397,26 \mathrm{C}+73.48963,80 \mathrm{D}-1197584,55 \mathrm{E}+2269516,3+\mathrm{F}=$ $=-322907,9$

$192010,01 \mathrm{C}+603179,96 \mathrm{D}+31797517,85 \mathrm{E}+21466039,52 \mathrm{~F}=$ $=70961,4$

$15328268,20 \mathrm{D}+20938517,5+\mathrm{E}-16351217,28 \mathrm{~F}=$ $=667165,27$

$47.4007330 .83 \mathrm{E}+815589.94 \quad \mathrm{~F}=$ $=916326,56$

$41810500,71 \mathrm{~F}=$ $=585929,77$

$81 \mathrm{~A}^{\prime}+8289,9 \mathrm{~B}^{\prime}+6.560,1 \quad \mathrm{C}^{\prime}+886923,61 \quad \mathrm{D}^{\prime}+730319,15 \quad \mathrm{E}^{\prime}+65.4992,53 \quad \mathrm{~F}^{\prime}=$ $=2739752$

$38498,40 \mathrm{BJ}^{\prime}-16397,26 \mathrm{C}^{\prime}+7348963,80 \mathrm{D}^{\prime}-1197584,55 \mathrm{E}^{\prime}+2269516,34 \mathrm{~F}^{\prime}=$ $=387590,0$ $192\left(1.40,01 \mathrm{C}^{\prime}+603.479,96 \quad \mathrm{D}^{\prime}+31797517,85 \mathrm{E}^{\prime}+21466039,52 \mathrm{~F}^{\prime}=\right.$ $=360 \cdot 10,3$

$15328268,20 \mathrm{D}^{\prime}+20938517,54 \mathrm{E}^{\prime}-16351217,28 \mathrm{~F}^{\prime}=$ $=1688303,70$ $47+407330,83 \mathrm{E}^{\prime}+815589,94 \mathrm{~F}^{\prime}=$ $=2387082,49$ $41810500,71 \mathrm{~F}^{\prime}=$ $=-61612,80$ sioni :

$$
\begin{array}{r}
H_{\mathrm{n}}=27104,1-20,536\left(\varphi-2160^{\prime}\right)-1,687 \lambda+0,05586\left(\varphi-2160^{\prime}\right)^{2}+ \\
+0,00191 \lambda^{2}+0,01400\left(\varphi-2160^{\prime}\right) \lambda \\
Z_{\mathrm{n}}=33708,4-9,444\left(\varphi-2160^{\prime}\right)-0,800 \lambda+0,10170\left(\varphi-2160^{\prime}\right)^{2}+ \\
+0,00503 \lambda^{2}-0,00147\left(\varphi-2160^{\prime}\right) \lambda
\end{array}
$$

che rappresentano la distribuzione normale delle due componenti nell'isola all'epoca 1935,0. In esse $\varphi$ è la latitudine geografica espressa in 
Tabella 1

\begin{tabular}{|c|c|c|c|c|}
\hline $\mathbf{N}$ & $\mathbf{F}$ & $Q$ & Numero & Località \\
\hline 1 & 248 & I & 1220 & San Vito (Capo) \\
\hline 2 & & 11 & 1221 & Giudaloca \\
\hline 3 & & III & 1222 & Borgo Annumziata \\
\hline 4 & & ) & 1223 & Pizzolungro \\
\hline 5 & 249 & I & 1224 & Faro di Capo Gallo \\
\hline 6 & & Il & 1225 & Case Olio di Lino \\
\hline $\bar{i}$ & & $\eta$ & 1226 & Luparello - R. I. Zoot. \\
\hline 8 & & 111 & 1227 & S. Cataldo \\
\hline 9 & & IV & 1228 & Portella Scaletti \\
\hline 10 & 250 & II & 1231 & C. Sacerdote \\
\hline 11 & & III & 1232 & Grotta Arnone \\
\hline 12 & 251 & II & 1233 & Casa Costantino \\
\hline 13 & & III & 1234 & S. Ambrourio \\
\hline 14 & & $\eta$ & 1235 & Cefalù \\
\hline 15 & 252 & I & 1236 & Capo Calava \\
\hline 16 & & II & 1237 & Ucria (R. Cullotta) \\
\hline 17 & & III & 1238 & Casa Oliveto \\
\hline 18 & 253 & I & 1239 & Tracoccia \\
\hline 19 & & $"$ & 1240 & Capo Milazzo \\
\hline 20 & & II & $12+1$ & Madonna delle Grazie \\
\hline 21 & & III & 1242 & Casa Malvi \\
\hline 22 & 256 & I & 1243 & Levanzo \\
\hline 23 & & $\eta$ & $12+4$ & Favignana \\
\hline 24 & & II & $12+5$ & Capo Lilibeo \\
\hline 25 & & III & 1248 & Marettimo \\
\hline 26 & 257 & I & 1250 & Contrada Roccazzedda \\
\hline 27 & & II & 1251 & La Piana \\
\hline 28 & & III & 1252 & Chelli Minore (\%. 146) \\
\hline 29 & & IV & 1253 & Contrada Misiliscemi \\
\hline 30 & 258 & I & 1254 & Mass. Lupotto \\
\hline 31 & & II & 1255 & Contrada Giardinello \\
\hline 32 & & $n$ & 1256 & S. Leoluca \\
\hline 33 & & III & 1257 & R. Paradiso \\
\hline 34 & & IV & 1258 & Rapitala \\
\hline 35 & 259 & I & 1259 & Cimitero di Cerda \\
\hline 36 & & II & 1260 & Cimitero \\
\hline 37 & & III & 1261 & Contrada Massara \\
\hline 38 & & $"$ & 1262 & Contrada Cassata \\
\hline 39 & 260 & I & 1263 & Villa Giulia \\
\hline 40 & & II & 1264 & Contrade Terrazza \\
\hline 41 & & III & 1265 & Chiesa SS.ma Trinità \\
\hline
\end{tabular}




\begin{tabular}{|c|c|c|c|c|}
\hline N. & F & Q & Numero & Località \\
\hline 42 & 260 & IV & 1266 & Cimitero \\
\hline 43 & 261 & I & 1267 & Casa Otaito \\
\hline 44 & & II & 1269 & Casa Longo Caguto \\
\hline 45 & & III & 1270 & M. Castelli \\
\hline 46 & & IV & 1271 & Casello Cicaldo \\
\hline 47 & 262 & I & 1272 & Forza d'Agro \\
\hline 48 & & IV & 1277 & Contrada Cozzo Schisina \\
\hline 49 & 265 & I & 1278 & La Volta \\
\hline 50 & & IV & 1281 & Torre dei Gesuiti \\
\hline 51 & & ” & 1282 & Villa S. Giovanni \\
\hline 52 & 266 & I & 1283 & Cimitero \\
\hline 53 & & II & 1284 & Abbeveratoio di Parrino \\
\hline 54 & & III & 1285 & Torre del Barone \\
\hline 55 & & IV & 1286 & R. Casena \\
\hline 56 & 267 & I & 1287 & Polizello \\
\hline 57 & & II & 1288 & R. Coda di Volpe \\
\hline 58 & & III & 1289 & Contrada Zammuto \\
\hline 59 & & IV & 1290 & R. Le Piane \\
\hline 60 & & ” & 1291 & Cimitero \\
\hline 61 & 268 & I & 1292 & Contrada Funnicheddu \\
\hline 62 & & II & 1293 & Casa Bannatella \\
\hline 63 & & III & 1294 & Contrada Mistegi \\
\hline 64 & & ") & 1295 & Contrada Bagno \\
\hline 65 & & IV & 1296 & Casa Cantoniera (q. 670) \\
\hline 66 & $2 \pi 1$ & I & 1309 & Cimitero di Camastra \\
\hline 67 & & II & 1310 & Piana di Ciavarello \\
\hline 68 & & $"$ & 1311 & Cimitero \\
\hline 69 & & IV & $13\lrcorner 2$ & Casa Restino \\
\hline 70 & & ” & 1313 & Scuola Agricoltura \\
\hline 71 & 272 & I & 1314 & Casa Cimiotta \\
\hline 72 & & II & 1318 & Contrada Priolo Sopr. \\
\hline 73 & & III & 1321 & Falconara \\
\hline 74 & & IV & 1322 & Casa le Schiette \\
\hline 75 & 273 & III & 1326 & Casa Cancellieri \\
\hline 76 & & 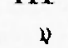 & 1328 & Contrada S.Nicolò delle Canne \\
\hline 77 & & ” & 1329 & Chiaromonte \\
\hline 78 & 275 & I & 1340 & Contrada Bèrdia \\
\hline 79 & & IV & 1346 & Contrada Fortugno \\
\hline 80 & & $"$ & 1347 & Contrada Manco \\
\hline 81 & 277 & I & 1350 & Mass. Li Greci \\
\hline
\end{tabular}


primi e $\lambda$ la longitudine geografica ugualmente espressa in primi a partire dal meridiano di Monte Mario.

Il controllo delle soluzioni dei sistemi [2] e [3] ha dato secondo notazioni evidenti:

$$
\begin{array}{ll}
{\left[l l_{\mathrm{i}}\right]_{\mathrm{H}}=4.2962} & {[v v]_{\mathrm{II}}=4.2896} \\
{\left[l l_{\mathrm{i}}\right]_{\mathrm{Z}}=178460} & {[v v]_{\mathrm{Z}}=178051}
\end{array}
$$

Sono stati calcolati quindi gnli errori medi della unità di peso per $\mathrm{i}$ quali sono stati ottenuti i valori sequenti:

$$
\mu_{\mathrm{H}}=23,9 \gamma \quad \mu_{\mathrm{z}}= \pm 48, \bar{\gamma} \gamma
$$

In base a cuesti valori mediante la relazione:

$$
m_{\mathrm{i}}=\frac{u}{\sqrt{[f f 5]_{\mathrm{x}_{\mathrm{i}}}}}
$$

sono stati calcolati $\mathrm{i}$ seguenti valori degli errori delle sei incognite per ognuno dei due sistemi [2] e [3].

$$
\begin{aligned}
& m_{\mathrm{A}}= \pm 28, \overline{5} \quad m_{\mathrm{B}}=1,668 \quad m_{\mathrm{C}}=0,463 \\
& m_{\mathrm{D}}=\leftarrow 0,00742 \quad m_{\mathrm{L}}=+0,00110 \quad m_{\mathrm{F}}=\div 0,00369 \\
& m_{\mathrm{A}}{ }^{\prime}=\therefore 58,2 \quad m_{13}{ }^{\prime}=+3,398 \quad m_{\mathrm{C}}{ }^{\prime}= \pm 0,914 \\
& m_{: !}^{\prime}= \pm 0.01513 \quad m_{!:}^{\prime}= \pm 0,00223 \quad m_{\mathrm{l}}^{\prime}= \pm 0,00752
\end{aligned}
$$

I valori [5] degli errori medi risultano dello stesso ordine di grandezza della precisione con cui è stata effettuata la misura della componente $H$ e calcolata la componente $Z$ secondo i dati di osservazione dell'Istituto Geografico Militare ( $\left.{ }^{2}\right)$.

Le [4] pertanto sono da ritenersi le espressioni più approssimate del "campo normale " di $H$ e $Z$ per la Sicilia per l'epoca 1935, 0 , compatibilmente con i dati di osservazione og̛gi disponibili.

La validità di dette espressioni del "campo normale " è hasata sulle seguenti considerazioni:

a) esse sono state dedotte dai dati magnetici più recenti e sistematici relativi alla sola Sicilia:

b) sono state calcolate utilizzando soltanto i valori non anomali di numerose stazioni uniformemente distribuite su tutta l'isola;

c) gli errori medi delle [4] sono dello stesso ordine degli er- 
ron i di cui sono affette le misure di $H$ e $Z$ della rete del $I^{\circ}$ ordine da cui sono tratte le espressioni stesse.

Dalle [4] si possono ottenere i seguenti gradienti normali di latitudine e di longitudine, utili nelle riduzioni per la correzione planetaria delle misure magnetiche del $2^{\circ}$ ordine:

$$
\begin{aligned}
& \frac{\partial I I}{\partial \varphi}=-20,536+0,11172\left(\varphi-2160^{\prime}\right)+0,01100 \lambda \\
& \frac{\partial H}{\partial \lambda}=-1,687+0,01400\left(\varphi-2160^{\prime}\right)+0,00382 \lambda \\
& \frac{\partial Z}{\partial \varphi}=-9,444+0,20340\left(\varphi-2160^{\prime}\right)-0,00147 \lambda \\
& \frac{\partial \eta}{\partial \lambda}=-0,800-0,00147\left(\varphi-2160^{\prime}\right)+0,01006 \lambda
\end{aligned}
$$

I sopradetti uradienti risultano espressi in $\gamma$ su primo e sono tabulati nelle tabelle n. $2,3,4,5$.

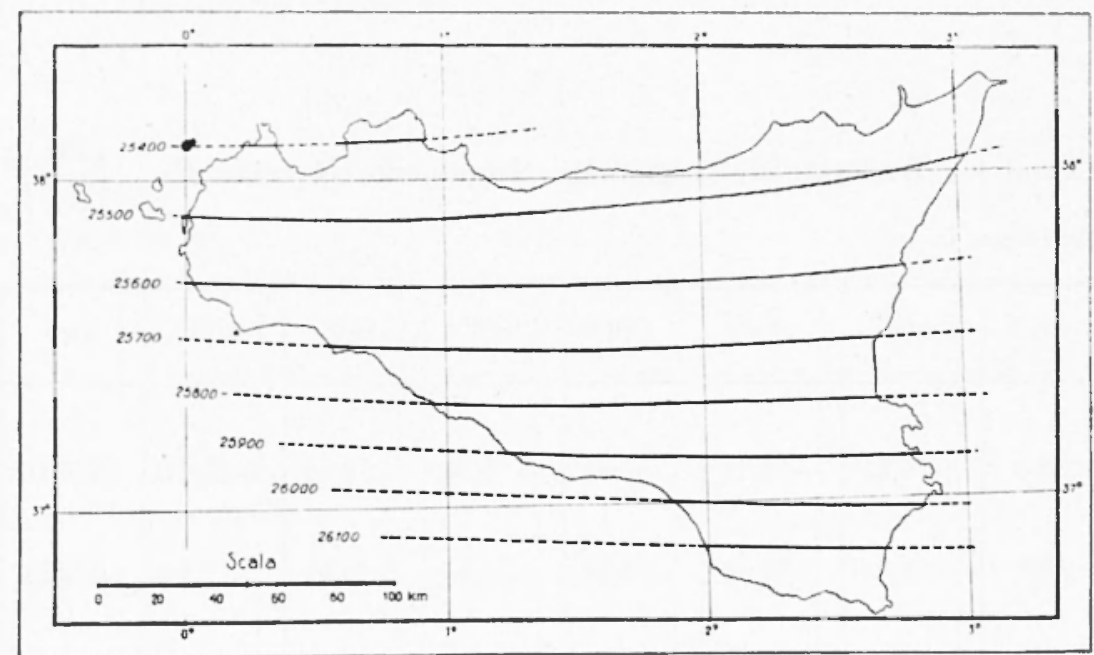

Fig. 1 - Campo normale per la componente $H$.

Nei grafici delle figg. 1 e 2 sono rappresentate rispettivamente le isodinamiche della $H$ e della $Z$ per l'intera isola; la fig. 3 dà invece la rappresentazione grafica dei gradienti di $H$ e $Z$ rispetto alle coordinate geografiche, espressi in $\gamma / \mathrm{km}$. 
TAB. 2 - Gradiente di lat. della componente $H$ verso $N:\left(\frac{\partial H}{\partial \varphi}\right)_{N}$ espresso in $\gamma /$;

\begin{tabular}{|c|c|c|c|c|c|c|c|}
\hline$\backslash$ & 00,0 & 00,5 & 10,0 & 10,5 & 20,0 & 20,5 & 30,0 \\
\hline 370,0 & $-13,83$ & $-13,41$ & $-12,99$ & $-12,57$ & $-12,15$ & $-11,73$ & $-1,31$ \\
370,5 & $-10,48$ & $-10,06$ & $-9,64$ & $-9,22$ & $-8,80$ & $-8,38$ & $-7,96$ \\
380,0 & $-7,13$ & $-6,71$ & $-6,29$ & $-5,87$ & $-5,45$ & $-5,03$ & $-4,61$ \\
380,5 & $-3,79$ & $-3,36$ & $-2,94$ & $-2,52$ & $-2,10$ & $-1,68$ & $-1,26$. \\
& & & & & & &
\end{tabular}

TAB. 3 - Gradiente di long. della componente $H$ verso $E:\left(\frac{\partial H}{\partial \lambda}\right)_{\mathrm{E}}$ espresso in $\gamma /$,

\begin{tabular}{|c|c|c|c|c|c|c|c|}
\hline${ }^{\lambda}$ & 00,0 & 00,5 & 10,0 & 10,5 & 20,0 & 20,5 & 30,0 \\
\hline 370,0 & $-0,85$ & $-0,73$ & $-0,62$ & $-0,50$ & $-0,39$ & $-0,27$ & $-0,16$ \\
\hline 370,5 & $-0,43$ & $-0,31$ & $-0,20$ & $-0,08$ & $+0,03$ & $+0,15$ & $+0,26$ \\
380,0 & $-0,01$ & $+0,11$ & $+0,22$ & $+0,34$ & $+0,45$ & $+0,57$ & $+0,68$ \\
380,5 & $+0,41$ & $+0,53$ & $+0,64$ & $+0,76$ & $+0,87$ & $+0,99$ & $+1,10$ \\
& & & & & & +
\end{tabular}


TABs. 4 - Gradiente di latit. della componente $Z$ verso $N:\left(\frac{\partial Z}{\partial \varphi}\right)_{N}$ espresso in $\gamma /$,

\begin{tabular}{|c|c|c|c|c|c|c|c|}
\hline$\backslash \lambda$ & 09,0 & 00,5 & 10,0 & 10,5 & 20,0 & 205 & $3^{0}, 0$ \\
\hline $37^{n}, 0$ & $\therefore 2,76$ & $+2,72$ & $+2,67$ & +2.63 & $\div 2,58$ & $+2,54$ & $+2,50$ \\
\hline 370,5 & $+8,86$ & $+8,82$ & $\div 8,77$ & $+8,3$ & $+8,69$ & $\because 8,64$ & $+8,60$ \\
\hline 380,0 & $\div 11,96$ & 11,92 & $+14,88$ & $1-14,83$ & $\div 14,79$ & $+14,74$ & $\div 14,70$ \\
\hline 380.5 & $+21,07$ & $+21,02$ & $+20,98$ & $-20,93$ & $+20,89$ & $+20,85$ & $+20,80$ \\
\hline
\end{tabular}

Tas. 5 - Gradiente di long. della componente $Z$ verso $E:\left(\frac{\partial Z}{\partial \lambda}\right)_{E}$ espresso in $\gamma /$,

\begin{tabular}{|c|c|c|c|c|c|c|c|}
\hline$\varphi$ & 00,0 & 00,5 & 10,0 & 10,5 & 20,0 & 20,5 & 30,0 \\
\hline 370,0 & $-0,89$ & $-0,9$ & $-0,28$ & $+0,02$ & $+0,32$ & $+0,62$ & $+0,92$ \\
370,5 & $-0,9 i$ & $-0,63$ & $-0,33$ & $-0,03$ & $+0,28$ & $+0,58$ & $+0,88$ \\
380,0 & $-0,98$ & $-0,67$ & $-0,37$ & $-0,07$ & $+0,23$ & $+0,53$ & $+0,84$ \\
380,5 & $-1,2$ & $-0,72$ & $-0,42$ & $-0,12$ & $+0,19$ & $+0,49$ & $\div 0,79$ \\
\hline
\end{tabular}


Misure assolute e variazione secolare media degli elementi magnetici in Sicilia. - In seguito alla esecuzione di una rete magnetica del $\mathrm{II}^{\circ}$ ordine nella Sicilia centro-settentrionale, avendo necessità di ancorare le misure relative, eseguite con bilance di Schmidt, ai valori assoluti delle componenti $H$ e $Z$, albiamo determinato nel marzo del 1954 i valori assoluti degli elementi $D, H$ e $I$ in cinque stazioni di cui 4 coincidenti con stazioni dell'I.G.M. essendo fra quest'ultime

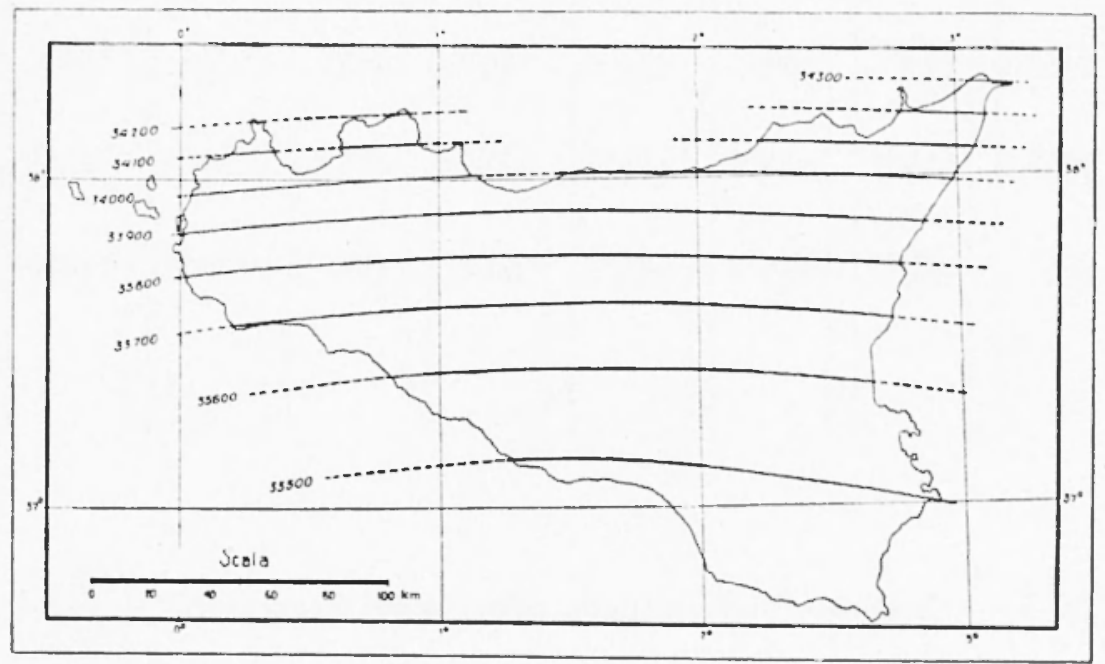

Fig. 2 - Campo normale per liı componente $Z$.

compresa la stazione fondamentale di Contrada Mistegi presso Caltanissetta.

L'attrezzatura strumentale di cui ci siamo serviti per detta campagna di misure assolute era la seguente:

a) un teodolite "Askania 》 da campo;

b) un induttore terrestre "Askania » da campo;

c) due magnetometri a torsione di La Cour (HTM) di fabluricazione "Askania »;

d) due bilance tipo Schmidt, per le componenti $I I$ e $Z$, per le determinazioni delle variazioni spaziali e temporali di $I I$ e $Z$ (le spa- 
ziali per il controllo delle variazioni da stazione a stazione, le temporali per la riduzione delle misure per la variazione diurna);
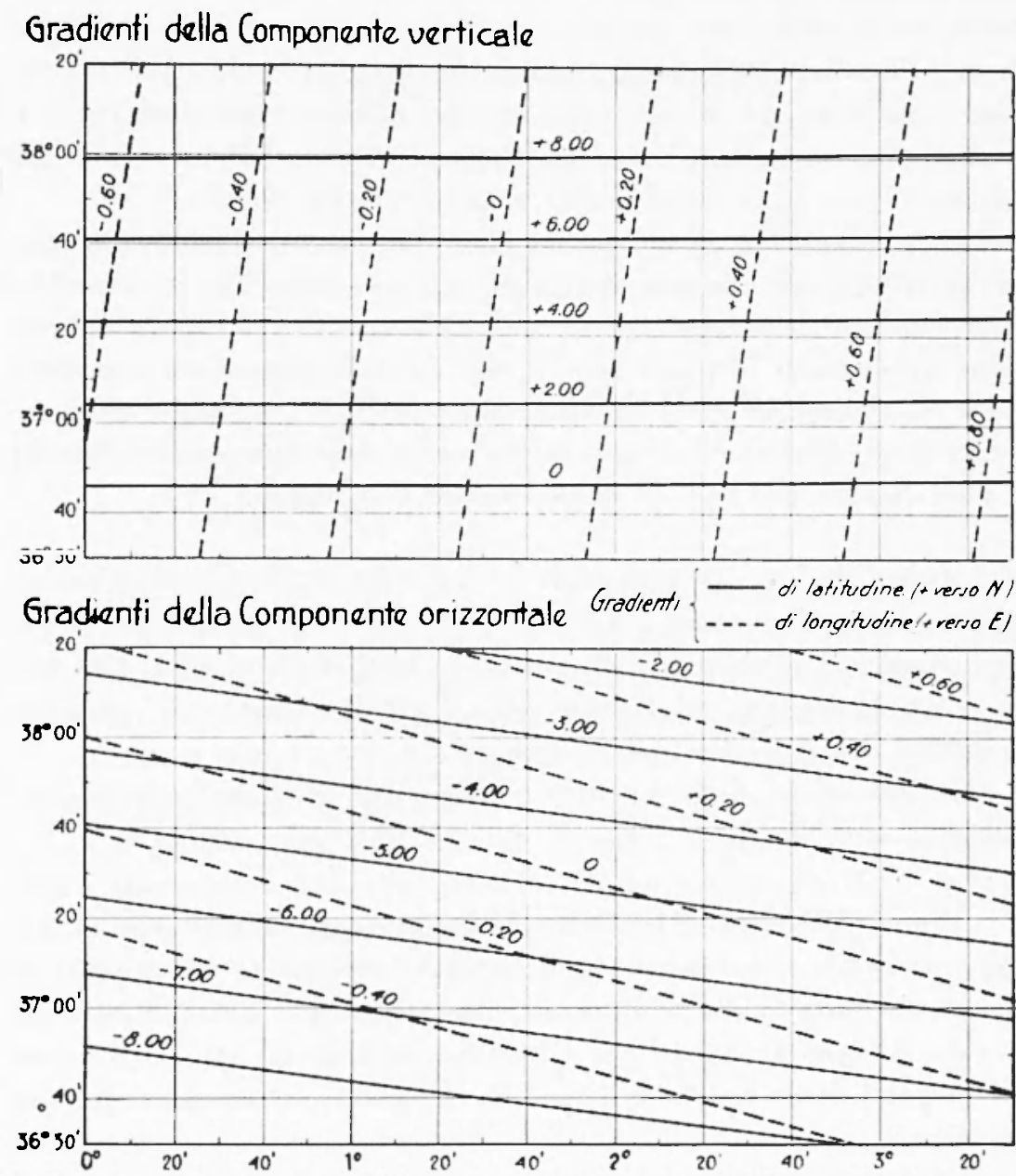

Fig. 3

e) un'apparecehiatura per la determinazione esatta del tempo costituita da: due cronometri tipo "Nardin ", un cronografo, un apparechio radio per la ricezione dei segnali ritmiei internazionali.

Le costanti strumentali dei magnetometri IITM come pure le caratteristiche gencrali delle bilance magnetiche sono riportate nella nota ( $\left.{ }^{1}\right)$ sopra citata. 
Misure di $H$. - Ie determinazioni assolute di $H$ sono state eseguite con entrambi i magnetometri a torsione, tarati nell'Osservatorio Mannetico di Fürstenfeldbruc's (Germania), allo scopo di avere un controllo delle misure ed anche una possibile compensazione nel caso di una eventuale leggera staratura dei magnetometri. In ogni stazione bono state fatte tre misure complete con ciascun mannetometro e si è attribuito a ciascuna stazione il valore medio delle due serie di misure.

E da notare che le misure eseguite con ciascun singolo magnetometro HTM sono risultate differenti generalmente di $1 \gamma$ e talvolta di $2 \gamma$; mentre le serie di misure relative a ciascuno dei due magnetometri differiscono di quasi $10 \gamma$ il che fareblue pensare ad una lieve staratura di uno dei due o di entrambi gli HTM. Comunque abbiamo attribuito a ciascuna stazione il valore medio delle due serie di letture; si può ritenere che l'errore di tale valore non superi i $5 \gamma$.

Misure di $I$. - Le misure di $I$ sono state eseguite con un induttore terrestre da campo "Askania) secondo le consuete norme di operazione con tale tipo di strumento. In ogni stazione sono state eseguite tre determinazioni distinte della $I$ mediante tre serie complete di misure con 8 letture per ciascuna serie.

La precisione delle misure ragriunta in ciascuna stazione è: $v_{\mathrm{I}}= \pm \mathrm{l}^{\prime}, 2$ ca.

Misura della $D$. - Le misure di $D$ sono state eseguite con il teodolite da campo "Askania ). La determinazione del meridiano magnetico è stata fatta mediante i magneti con sospensione a filo. Sono state eseguite 4 serie di misure per ciascuna posizione diritta e rovesciata dei magneti forte e debole aventi un rapporto dei momenti magnetici $K=2$.

La determinazione del nord geografico è stata esegruita in ogni stazione almeno 5 volte, mediante osservazioni di sole. L'esatta determinazione del tempo, quale è stata possibile con l'attrezzatura strumentale di cui era dotata la squadra di lavoro, ha consentito una precisione superiore al necessario.

Comunque la precisione finale della misura della $D$ in ogni stazione ha il valore: $v_{D}=+0^{\prime}, 7$.

Il valore assoluto della componente $Z$ è stato calcolato mediante la relazione: $Z=H \operatorname{tg} I$. 
La precisione dei valori della $Z$, tenuto conto dei valori della precisione con cui sono stati determinati i valori di $H$ e $I$, risulta pertanto:

$$
v_{\mathrm{Z}}=\left\lceil\left(\frac{\partial Z}{\partial H}\right)^{2} \nu^{2}{ }_{\mathrm{H}}+\left(\frac{\partial Z}{\partial I}\right)^{2} \nu_{\mathrm{I}}^{2}\right]^{1 / 2}
$$

che per i valori medi : $H_{\mathrm{m}}=25800 \gamma$ e $I_{\mathrm{m}}=53^{\circ}$ da $\gamma_{\mathrm{z}}=+25 \gamma$.

Per il calcolo della $Z$ i valori delle misure di $H$ e di $I$, fatte in ore diverse, sono stati ridotti allo stesso istante mediante le osservazioni delle variazioni temporali di $H$ e $Z$ eseguite con le due bilance di Schmidt che servivano inoltre al controllo delle variazioni locali tra una stazione e l'altra.

Le bilance di Schmidt, tarate all'inizio della campagna, avevano le seguenti costanti di scala:

$$
\varepsilon_{\mathrm{H}}=8,5+0,04 \gamma / \text { div. } \quad \varepsilon_{\mathrm{z}}=11,30-0,05 \gamma / \text { div } .
$$

Tutti i valori assoluti di $I, I I, D, Z$ sono stati ridotti poi ad un istante determinato e precisamente alle ore $0^{\text {h }}$ del giorno 28 marzo 1954, che è stato scelto in quanto l'andamento delle variazioni degli elementi magnetici corrispondeva a condizioni magneticamente calme.

I valori definitivi delle stazioni assolute osservate si riferiscono pertanto all'istante suddetto.

Per la riduzione delle misure all'epoca sopradetta ci siamo serviti delle registrazioni variografiche degli elementi $D, I I, Z$ ottenute con i variografi "Ruska” dell'Osservatorio di Gihilmanna.

La taratura di detti variografi eseguita all'inizio della campagna ha fornito i seguenti valori per le costanti di scala dei tre strumenti:

$$
\begin{aligned}
& \varepsilon_{1 \mathrm{II}}=2,71 \div 0,06 \gamma / \mathrm{mm} \\
& \varepsilon_{\mathrm{p}}=0,98 \div 0.02 \% \mathrm{~mm} \\
& \varepsilon_{\mathrm{z}}=7,30 \therefore 0,04 \gamma / \mathrm{mm}
\end{aligned}
$$

I dati topografici ed i valori assoluti degli elementi magnetici osservati e calcolati, per ciascuna delle stazioni in cui sono state eseguite le misure, sono contenuti nella tabella $n .6$, in cui manca il ralore della inclinazione della stazione n. 3 non misurata per condizioni metcorologiche avverse.

La tabella n. 7 contiene invece per le quattro stazioni assolute, coincidenti con quelle dell'I.G.M. compresavi la stazione fondamentale 
Tabella 6

\begin{tabular}{|c|c|c|c|c|c|c|c|c|c|c|c|c|}
\hline Staz. & $\begin{array}{c}n . \\
\text { IGM }\end{array}$ & Localitì & $\uparrow$ Nord & 2. Est & $\begin{array}{c}\text { alt. in } \\
\mathrm{m}\end{array}$ & $\begin{array}{l}\text { Data di } \\
\text { osserv. } \\
\text { di H }\end{array}$ & II $\mathrm{aga}$ & $\begin{array}{l}\text { Data di } \\
\text { osserv. } \\
\text { di I }\end{array}$ & Ious & $\begin{array}{c}\text { Data di } \\
\text { osserv. } \\
\text { di ID }\end{array}$ & Dовя & Zcalc. \\
\hline 1 & - & $\begin{array}{l}\text { Osservatorio di } \\
\text { Gibilmanna }\end{array}$ & $37^{0} 59^{\prime} 13^{\prime \prime}$ & $1 " 3+21 "$ & 975 & $\begin{array}{l}21.3 .54 \\
15^{\mathrm{ht}} 10^{\mathrm{m}}\end{array}$ & 25821 & $\begin{array}{l}21.3 .5 .4 \\
16^{\mathrm{l}} 00^{\mathrm{m}}\end{array}$ & $53^{\prime \prime 28}, 9$ & $\begin{array}{l}21.3 .5 .4 \\
13^{\mathrm{n}} 08^{\mathrm{m}}\end{array}$ & $-2^{\prime \prime} 11^{\prime}, 2$ & 31872 \\
\hline 2 & 1294 & $\begin{array}{l}\text { Contrada Mistegi } \\
\text { (Caltanissetta) }\end{array}$ & $37^{\prime \prime 26} 19^{\prime \prime}$ & $1^{\circ} 35^{\circ} 49^{\prime \prime}$ & 385 & $\begin{array}{l}22.3 .54 \\
10^{\mathrm{ln}} 59^{\mathrm{m}}\end{array}$ & 26066 & $\begin{array}{l}22.3 .54 \\
12^{\mathrm{h}} 18^{\mathrm{m}}\end{array}$ & $52^{\prime \prime} 51^{\prime}, 2$ & $\begin{array}{l}22.3 .54 \\
9^{\mathrm{h}} 35^{\mathrm{m}}\end{array}$ & $-2^{0}\left(03^{\prime}, 9\right.$ & 31.122 \\
\hline 3 & 1264 & $\begin{array}{l}\text { C.e } \\
\text { (Sperrazza } \\
\text { Speringa) }\end{array}$ & $37^{0.45} 14^{\prime \prime}$ & $105122 "$ & 672 & $\begin{array}{l}23.3 .54 \\
12^{11} 27^{\mathrm{m}}\end{array}$ & 25854 & - & - & $\begin{array}{l}23.3 .54 \\
11^{1 \mathrm{t}} 58^{\mathrm{m}}\end{array}$ & $-2^{0}(12 ; 0$ & - \\
\hline 4 & 1262 & $\begin{array}{l}\text { C.a Cassata } \\
\text { (Ventimiglia) }\end{array}$ & $37^{\prime \prime} 54 \cdot 45^{\prime \prime}$ & $1^{\prime \prime} 0632^{\prime \prime}$ & 402 & $\begin{array}{l}24.3 .54 \\
9^{11} 29^{\mathrm{m}}\end{array}$ & 25808 & $\begin{array}{l}24.3 .54 \\
10^{\mathrm{h}} 33^{\mathrm{m}}\end{array}$ & $53^{0} 24^{\prime}, 4$ & $\begin{array}{l}24.354 \\
11^{\mathrm{h}} 22^{\mathrm{m}}\end{array}$ & $-2^{0} 19^{\prime}, 7$ & 31762 \\
\hline 5 & 1232 & $\begin{array}{l}\text { Grotta Agnone } \\
\text { (Cilpo Zafferano) }\end{array}$ & $38^{\circ} 06^{\prime} 13^{\prime \prime}$ & $1^{10} 0+56 "$ & 12 & $\begin{array}{l}24.3 .5 .4 \\
17^{11} 14^{\mathrm{m}}\end{array}$ & 257.16 & $\begin{array}{l}24.3 .54 \\
17^{\mathrm{h}} 44^{\mathrm{m}}\end{array}$ & $53^{\prime \prime} 36^{\prime}, 4$ & $\begin{array}{l}24.3 .54 \\
16^{\mathrm{h}}+7^{\mathrm{m}}\end{array}$ & $--2^{\prime \prime} 09^{\prime}, 8$ & 34929 \\
\hline
\end{tabular}

TABILLLA 7

\begin{tabular}{|c|c|c|c|c|c|c|c|c|c|c|c|c|c|c|c|c|}
\hline $\begin{array}{c}1 \\
\text { Sta\%. } \\
\text { I.N.G. } \\
\text { n. }\end{array}$ & $\left|\begin{array}{c}2 \\
\text { Staz. } \\
\text { I.G.M. } \\
n .\end{array}\right|$ & $\begin{array}{c}3 \\
\text { Понн } \\
\gamma\end{array}$ & $\begin{array}{c}4 \\
\text { Ridluz. } \\
\text { al } \\
1954.25 \\
\text { Jil }\end{array}$ & $\begin{array}{c}5 \\
\text { II } \\
195.4 .25\end{array}$ & $\mid$\begin{tabular}{c|}
6 \\
\\
II \\
1935.0
\end{tabular} & 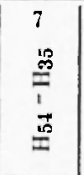 & $z^{8}$ & $\begin{array}{c}9 \\
\text { Riduz. } \\
\text { al } \\
1954,25 \\
د Z\end{array}$ & $\begin{array}{c}10 \\
7 \\
1954.25\end{array}$ & $\begin{array}{c}11 \\
Z \\
1935.0\end{array}$ & $\underbrace{12}$ & $\begin{array}{c}13 \\
\mathrm{D}_{\mathrm{OBB}}\end{array}$ & $\begin{array}{c}14 \\
\text { Riduz. al } \\
195.4 .25 \\
\text { JD }\end{array}$ & $\begin{array}{c}15 \\
17 \\
1954.25\end{array}$ & $\begin{array}{c}16 \\
10 \\
1935\end{array}$ & $\begin{array}{l}17 \\
18 \\
0 \\
0 \\
\vdots \\
0 \\
0\end{array}$ \\
\hline 2 & 129.4 & 26066 & +13 & 26079 & $2 ; 7.18$ & -331 & 3.1422 & -24 & 34446 & 33657 & $\div 789$ & $-2003^{1}, 9$ & $-l^{\prime}, 5$ & $-2005^{\prime}, 4$ & $-4021^{1}, 4$ & $+2^{\circ} 16^{\prime} .0$ \\
\hline 3 & 1264 & 25854 & +12 & 25866 & 25521 & -3.15 & - & - & - & - & - & $-2^{00} 02^{\prime}, 6$ & $\div 5^{\prime}, 2$ & -1057 & 一.1016',6 & $+201{ }^{\prime}, 2$ \\
\hline 4 & 1262 & 25808 & +31 & 25842 & 25.181 & +-361 & 3.462 & -6 & 3.1768 & 33979 & $-1-789$ & $-2019^{\prime}, 7$ & $+3,3$ & $-2016^{\prime}, 4$ & $-4038^{\prime}, 1$ & $+2021^{1} .7$ \\
\hline 5 & 1232 & 257.16 & +11 & 25757 & $25 \div 26$ & -331 & 31929 & -2 & 34927 & 3.1132 & +795 & $-2^{00} 09^{\prime}, 8$ & $+2^{\prime}, 0$ & $-2^{0007}, 8$ & $-4^{0} 36^{\prime}, 3$ & $+2028,5$ \\
\hline
\end{tabular}


n. 1294 di Contrada Mistegi (Caltanissetta), le riduzioni dei valori osservati alle ore $0^{\text {h }}$ del 28 marzo 1954, i corrispondenti valori dell'J.G.M. ridotti al 1935,0 e le differenze dei valori assoluti tra le due epoche.

Mediante le differenze tra i valori degli elementi $D, H$ e $Z$, alle epoche 1935,0 e 1954,25 riportati nelle colonne $7^{\mathrm{a}}, 12^{\mathrm{a}}$ e $17^{\mathrm{a}}$ della tabella n. 7, abbiamo computato la variazione secolare media degli stessi elementi per gli anni compresi tra le epoche stesse.

Si può notare che le differenze dei valori della $H$ fra le due epoche sono molto concordanti e danno un valore medio: $\Delta H_{\mathrm{m}}=342 \%$.

Tabella 8

\begin{tabular}{|c|c|c|c|}
\hline \multirow{2}{*}{ Comp. } & \multicolumn{2}{|c|}{$\begin{array}{c}\text { V. S. } \\
\text { (Vestine e collal,.) }\end{array}$} & \multirow{2}{*}{$\begin{array}{l}\text { v. s. m. oss. } \\
\text { tra le epoche } \\
1935,0-1954,5\end{array}$} \\
\hline & 1932,5 & 1942,5 & \\
\hline D & $\div 8,9 \quad 1 / a$ & $+7,6 \quad 1 / a$ & $\div 7,2 \quad 1 / a$ \\
\hline II & $\div 12,5 \quad \gamma / u$ & $+30,7 \quad \gamma / a$ & $+17,8 \quad \gamma / a$ \\
\hline $\mathrm{Z}$ & +22.9 & $+39,9$ & $+41,2$ \\
\hline
\end{tabular}

Gli scarti delle singole differenze dalla media risultano inferiori all'errore medio di $H$ 1935,0.

Anche le differenze tra i valori della $Z$ nelle due epoche sono in ottimo accordo; la media delle differenze è:

$$
\Delta Z_{\mathrm{m}}=791 \% \text {. }
$$

e lo scarto massimo delle singole differenze dal valore medio ¿̀ di appena $4 \gamma$, trascurabile rispetto alla precisione delle misure di $Z$.

I valori delle analoghe differenze della $D$ nelle due epoche sono ugrualmente molto concordanti; il loro valore medio: $D_{\mathrm{m}}=+2^{\circ} 20^{\prime}, 6$ con uno scarto massimo di $7^{\prime}, 9$, dalla media.

In base a tali valori medi delle differenze è stata computata la variazione secolare media nell'intervallo 1935,0-1954,25 per i tre elementi $D, H, Z$; i rispettivi valori si trovano nella tabella n. 8. Nella stessa tabella abbiamo pure riportate la v. s. dei tre elementi $D, H, Z$, 
calcolata, per la Sicilia centro-settentrionale, mediante i valori planetari delle tabelle per la v. s. negli anni 1932,5 e 1942,5 di Vestine e collab. della Carnegie Institution $(3)$.

Dal confronto dei valori di detta tabella e dalla considerazione della tendenza delle isopore al 1942.5 si trae una soddisfacente conferma della attendibilità dei valori trovati per detta regione.

Data la grande precisione con cui possono determinarsi i valori assoluti di $I I$ e $Z$ mediante magnetometri a filo di quarzo $H Z M$ e $H T M$ si spera che le future campagne per la ripetizione della rete nazionale del $1^{\circ}$ ordine possano eseguirsi con tali strumenti i quali peró naturalmente hanno bisogno di periodiche tarature di controllo da farsi presso un Osservatorio Magnetico.

Roma - Istituto Nazionale di Geofisica -- Aprile 1954

\section{RIASSUNTO}

Fiene determinato il "campo normale» al 1935,0 delle componenti H e Z del campo magnetico terrestre, valuvole per la Sicilia.

Sono state calcolate espressioni di $\mathrm{II}^{\circ}$ grado nelle coordinate geografiche applicando il metodo dei minimi quadrati ai valori assoluti forniti dall Istituto Geografico Militare.

Viene discussa la validita di queste espressioni. Iengono inoltre forniti $i$ valori dei gradienti normali di latitudine e longitudine delle due componenti.

Sulla base dei dati di osservazione ottenuti in una campagna di misure assolute effettuate in stazioni gia note, viene computata la variazione secolare media degli elementi D. II e Z tra gli anni 1935-5\%.

\section{$S L M M A R Y$}

The "normal field" referred to 1935-0 of $\mathrm{H}$ and $\mathrm{Z}$ components of the Earth's magnetic field, valid for Sicily is determined.

II degree expressions have been calculated in geographycal coordinates by applying the method of least squares relating to absolute values supplied by Italian Military Geographycal Institute.

The validity of these expressions is illustrated. Furthermore the 
values of the normal gradientes of latitude and longitude of $\mathrm{If}$ and $\mathrm{Z}$ are supplied.

On the basis of the observational data, obtained during a survey of absolute measurements, carried out on already hinoun stations, average secular variations of elements $\mathrm{D}, \mathrm{H}$ and $\mathrm{Z}$ for 1935 and 1954 are computed.

\section{BIBLIOGRAFIA}

1) M. Gionoi, E. MrDI, F. MoLisi, Rilievo magnetico del ll ordine della Sicilia centro-settentrionale. Annali di Geofisica, a. VII, 1954.

(-) C. Monet.1.I, La rete geofisica e geodetica in Italia nel suo stato attuale. Trieste, 1948.

(3) Vestixe E. H., Laponte L., Lavge I., Cooper C., Hexprax W. C., Descriplion of the Earth's Main Magnetic Field and its Secular Change, 1905-1945. Dep.t Terr. Magn. Carnegie Institution Washington 1917. 